Una revisión del estado de conservación

de los mamíferos australianos

\title{
A review of the conservation status of Australian mammals
}

\author{
John C. Z. Woinarski ${ }^{1 *}$, Andrew A. Burbidge ${ }^{2}$, and Peter L. Harrison ${ }^{3}$
}

\begin{abstract}
'National Environmental Research Program North Australia and Threatened Species Recovery Hub of the National Environmental Science Programme, Charles Darwin University, NT 0909. Australia. E-mail: john.woinarski@cdu.edu.au (JCZW)

${ }^{2}$ Western Australian Wildlife Research Centre, Department of Parks and Wildlife, PO Box 51, Wanneroo, WA 6946, Australia. E-mail: amburbidge@westnet.com.au (AAB)

${ }^{3}$ Marine Ecology Research Centre, School of Environment, Science and Engineering, Southern Cross University, PO Box 157, Lismore, NSW 2480, Australia. E-mail: Peter.Harrison@scu.edu.au (PLH)

${ }^{*}$ Corresponding author
\end{abstract}

\begin{abstract}
Introduction: This paper provides a summary of results from a recent comprehensive review of the conservation status of all Australian land and marine mammal species and subspecies. Since the landmark date of European settlement of Australia (1788), at least 28 of the ca. 272 Australian endemic land mammal species have been rendered extinct.

Results and Discussion: Extinctions have occurred at a more or less consistent rate of one to two species per decade since the 1840s, with that rate continuing unabated. A further 55 species from that original fauna are now threatened, and an additional 42 are Near Threatened. Although many factors have contributed to these declines and extinctions, and the array of threats varies amongst individual species, the threat that has had (and is continuing to have) most detrimental impact upon terrestrial mammal species is predation by the introduced cat Felis catus and European red fox Vulpes vulpes. There has been some successful broad-scale management of the fox, but the threat posed by feral cats remains largely unabated. For the 55 species occurring in Australian marine waters, the information base is mostly too meagre to assess conservation status other than as Data Deficient. For the Australian mammal fauna generally, the current conservation management effort is insufficient, with ongoing trends for decline in many species - for example, of 49 species whose conservation status changed over the period 1992-2012, 38 had deteriorating conservation status whereas only 11 had improving status.
\end{abstract}

Key words: extinct, feral cat, fire, marine mammals, marsupial, predation, red fox, threatened, translocation.

\section{Introduction}

Australia's mammal fauna is rich and extremely distinctive (Holt et al. 2013). It is also diminishing very rapidly (Burbidge and McKenzie 1989; Burbidge et al. 2008; McKenzie et al. 2007; Woinarski et al. 2011). Recognising conservation concern about that trend, we recently undertook the first comprehensive review of the conservation status of the Australian mammal fauna (all terrestrial and marine species and subspecies in Australia, its external territories and territorial waters) (Woinarski et al. 2014). In this paper, we summarise some of the main findings of that review.

As Australia is a nation occupying an entire continent, such a national conservation overview is appropriate and can be related readily to national conservation management activity and legislation. We recognise that the conservation status of Australian mammal species has been assessed recently by the IUCN as part of a broader global review (Hoffmann et al. 2011; Schipper et al. 2008), however that broad assessment has had little consequence for the conservation of Australian mammal species - for example, the global review resulted in no changes in the listing of threatened species under Australian national legislation, and provided no substantial management 
advice for the conservation of Australia's threatened mammals. Our objectives were: to provide an explicit focus on the conservation of Australian mammals; to provide information in a manner that would be most appropriately formatted for overhaul of the now somewhat sub-optimal listing of threatened species under Australian legislation; to assess the extent to which current conservation management actions were delivering benefits to Australia's threatened mammals; to identify major factors affecting the status of Australian mammals; and to identify research and management priorities.

The approach we adopted was modelled on comparable overviews of Australian birds, that sought to provide not only an assessment of the conservation status of all taxa but also to frame and guide a coherent conservation management response (Garnett and Crowley 2000; Garnett et al. 2011). Our review was also substantially informed by now somewhat dated conservation assessments for particular groups of Australian mammals, including marsupials (Maxwell et al. 1996), rodents (Lee 1995), bats (Duncan et al. 1999), cetaceans (Bannister et al. 1996) and seals (Shaughnessy 1999).

More so than most other areas, the status of Australian mammals can be benchmarked to a pivotal historical event, the first settlement of Australia by Europeans in 1788. Although a rich component of the Australian mammal fauna was lost before this date-most notably the extirpation of the extraordinary Australian megafauna over the period from about 60,000 to 20,000 years ago (Johnson 2006) - European settlement saw a marked environmental transformation across many areas of Australia (Bradshaw 2012) and the introduction of very many plant and animal species whose environmental impacts have been profound. Accordingly, we use 1788 as a baseline from which to chart the conservation fate of the Australian mammal fauna.

\section{Material and methods}

Our objective was to assess the conservation status of all Australian mammal taxa, using IUCN criteria (IUCN 2001). These criteria mostly relate to distributional extent, and population size and trends. Accordingly, we required an inventory of all Australian mammal taxa, and information on the distribution and population of those taxa.

The taxonomy of Australian mammals is unsettled, with at least 50 endemic species described since 1970. We established a checklist of species and subspecies recorded in Australia and its external territories, based on consensus among a group of taxonomists working actively with Australian mammals, and in part informed by a far more comprehensive taxonomic treatment of the Australian mammal fauna in preparation (Jackson and Groves in press). As evidence of the fluid nature of this taxonomy, six species of Australian endemic mammals (three bats (Mormopterus spp.) and three dasyurids (Antechinus spp.)) and a nearly-endemic coastal dolphin, Sousa sahulensis, have been described since 2012 (Baker et al. 2013; Baker et al. 2014; Baker et al. 2012; Jefferson and Rosenbaum 2014; Reardon et al. 2014). The new Mormopterus species and the Sousa species were included in our review, but the new Antechinus were not considered, because their description post-dated our assessments.

There is no comprehensive distributional or monitoring data base for Australian mammals, although The Atlas of Living Australia (http://www.ala.org.au/) now provides a reasonable and increasingly comprehensive collation of distributional records. For our review, we collated distributional records for all terrestrial Australian mammal taxa from a wide range of state conservation agency and museum sources. This database used as a foundation a previous collation of mainland non-volant mammal records developed by Fisher et al. (2014), and added to it records of bats and Tasmanian mammals, from relevant data bases maintained by state agencies 
and museums. All resulting maps were inspected for outlying and potentially erroneous records, with any such records then referred back to the original source for confirmation or rejection. Subsequently, we used recent (post 1992) records to calculate area of occupancy and extent of occurrence, following IUCN Standards and Petitions Subcommittee (2013). Distributional data for marine species were too meagre to allow for such analysis.

Reliable estimates of population size are available for only a few Australian mammal species (mostly large and commercially exploited kangaroo species, a few whale species, and a small set of highly localised threatened species). However, there is some information on trends in various measures of relative abundance for more species.

Based on this compilation of relevant conservation parameters, we assessed all taxa using IUCN criteria, as either Extinct, Critically Endangered (Possibly Extinct), Critically Endangered, Endangered, Vulnerable, Near Threatened, Least Concern or Data Deficient. We allocated some taxa to Near Threatened (Conservation Dependent), a category no longer used by IUCN, but which is available under Australian legislation. These assessments related only to the Australian range of (the minority of) species that were not Australian endemics. This draft assessment and a detailed account were then widely distributed to additional relevant experts, and the assessments and accounts were reviewed in response to feedback. More than 200 experts provided such reviews. We used a cut-off date of December 2012 for all assessments, and also provided retrospective assessments (based on currently available information) of the conservation status of all taxa 10 and 20 years earlier. For extinct species, we documented the most recent record, and estimated the decade of extinction.

In addition to compiling information on these conservation parameters, we documented information on threatening factors for all taxa assessed as threatened or Near Threatened, rating threats according to the extent over which they operated (in relation to the distribution of the considered species), the intensity of their impact, and whether the threats were historical, current or projected.

\section{Results}

There is a marked distinction between the conservation status of Australian terrestrial and marine mammals (Table 1). Of 272 terrestrial Australian-endemic mammal species extant in 1788, we considered 28 are now extinct (Tables 1, 2). A recent unsuccessful search for the highly localised (known only from a 5 ha cay) Bramble Cay melomys Melomys rubicola suggests that the number of extinctions is now 29, with that loss sometime since its last record, in 2009. In addition, the Christmas Island shrew Crocidura trichura was considered Critically Endangered (Possibly Extinct): with no records since 1985 , it is likely now also to be extinct. Hence, in the 236 years since initial European settlement of Australia, more than $10 \%$ of the endemic land mammal fauna has been rendered extinct. Our assessment concluded that the first post-1788 extinction was around the 1840 s, and that the rate of extinction (of 1-2 species per decade) has continued unabated since then, with two species lost in the last 10 years (the Bramble Cay melomys and Christmas Island pipistrelle Pipistrellus murrayi).

Our review assessed a further 55 Australian endemic land mammals as threatened (i.e. Critically Endangered, Endangered or Vulnerable), and a further 42 species as Near Threatened - i.e. $46 \%$ of the Australian endemic terrestrial mammal species present in 1788 is now extinct, threatened or Near Threatened (Tables 1, 2). The dominant trend for the threatened and Near Threatened species (for which such information was available) was for ongoing decline (with 38 terrestrial mammal species considered to have worsened conservation status over the period 1992 to 2012 
and 11 species showing an improved conservation status over this period). Many species are now reduced to very small population sizes (e.g. nine species now have populations of fewer than 1000 individuals), or ranges that are now a minute proportion of their former distributions.

As recognised previously (Burbidge 1999), islands have made a notable contribution to the conservation of Australia's mammals: seven species that have been lost from all of their former extensive mainland ranges have survived solely because subpopulations have persisted on continental islands. However, the fate of Australian mammals restricted to oceanic islands has been far less good: for these six species (five species endemic to Christmas Island and one to Lord Howe Island), four are now extinct, one is Critically Endangered (Possibly Extinct) and the

Table 1. Tallies for the Australian conservation status of mammal taxa occurring in Australia. Conservation status codes: EX extinct; $\mathrm{CR}(\mathrm{PE})$ Critically Endangered (Possibly Extinct); CR Critically Endangered; EN Endangered; VU Vulnerable, NT(CD) Near Threatened (Conservation Dependent), NT Near Threatened; LC Least Concern; DD Data Deficient.

\begin{tabular}{|c|c|c|c|c|c|c|c|c|c|c|c|c|}
\hline \multirow[t]{2}{*}{ Taxa considered } & \multirow{2}{*}{$\begin{array}{l}\text { No. of } \\
\text { taxa }\end{array}$} & \multicolumn{9}{|c|}{ Status assigned } & \multirow{2}{*}{$\begin{array}{c}\% \text { extinct and } \\
\text { threatened }\end{array}$} & \multirow{2}{*}{$\begin{array}{c}\% \text { extinct and } \\
\text { threatened }\end{array}$} \\
\hline & & EX & $\begin{array}{l}\text { CR } \\
\text { (PE) }\end{array}$ & CR & EN & VU & $\begin{array}{l}\text { NT } \\
\text { (CD) }\end{array}$ & NT & LC & DD & & \\
\hline \multicolumn{13}{|c|}{ All species } \\
\hline terrestrial species & 315 & 29 & 2 & 9 & 10 & 36 & 5 & 47 & 175 & 2 & 27.3 & 27.5 \\
\hline marine species & 58 & 0 & 0 & 0 & 4 & 2 & 0 & 5 & 12 & 35 & 10.3 & 26.1 \\
\hline total all species & 373 & 29 & 2 & 9 & 14 & 38 & 5 & 52 & 189 & 37 & 24.7 & 24.8 \\
\hline \multicolumn{13}{|c|}{ Endemic species } \\
\hline terrestrial species & 272 & 28 & 2 & 9 & 10 & 34 & 4 & 38 & 145 & 2 & 30.5 & 30.7 \\
\hline marine species & 1 & 0 & 0 & 0 & 0 & 1 & 0 & 0 & 0 & 0 & 100 & 100 \\
\hline total all species & 273 & 28 & 2 & 9 & 10 & 35 & 4 & 38 & 145 & 2 & 30.8 & 31.0 \\
\hline \multicolumn{13}{|c|}{ All species and subspecies } \\
\hline terrestrial & 440 & 36 & 3 & 9 & 21 & 57 & 8 & 63 & 239 & 4 & 28.6 & 28.9 \\
\hline marine & 62 & 0 & 0 & 1 & 4 & 2 & 0 & 5 & 12 & 38 & 11.3 & 29.2 \\
\hline total all species & 502 & 36 & 3 & 10 & 25 & 59 & 8 & 68 & 251 & 42 & 26.5 & 28.9 \\
\hline
\end{tabular}

remaining species (the Christmas Island Flying-fox Pteropus natalis) is Critically Endangered.

Also, as recognised previously, the fate of Australian mammals has varied between different taxonomic groups, sizes, ecologies and distributions (Burbidge and McKenzie 1989; Burbidge et al. 2009; Cardillo and Bromham 2001; Chisholm and Taylor 2007; Fisher et al. 2014; Johnson and Isaac 2009; Murphy and Davies 2014). Medium-sized (ca. $35 \mathrm{~g}$ to $5.5 \mathrm{~kg}$ ) mammals (such as larger dasyurids, larger rodents, smaller macropods, potoroids and bandicoots) have been most affected, arboreal species less affected, and declines and extinctions have occurred mostly in semi-arid, arid and temperate areas, with notable chronological variation. Previously considered relatively stable, the mammal fauna of much of northern Australia is now undergoing rapid and severe decline (Woinarski et al. 2011).

Although individual species have differed in the pattern and cause of their decline (or extinction), and many factors may have contributed, the factor that has contributed (and continues to contribute) most to declines for terrestrial species has been predation by two introduced species, the European red fox Vulpes vulpes and feral cat Felis catus (Woinarski et al. 2014). Other major factors implicated 
Table 2. List of Australian mammal species assessed as extinct, threatened or Near Threatened in current review (MAP = Mammal Action Plan), ordered by conservation status. The conservation status (as at 2012) according to Australian legislation (EPBCA) and IUCN Red List is also given. Note that the Australian listing does not include a Near Threatened category. For MAP and EPBCA, the conservation status assigned relates (for non-endemic species) to only that part of their population in Australia or Australian waters. Conservation status: EX Extinct, CR(PE) Critically Endangered (Possibly Extinct), CR Critically Endangered, EN Endangered, VU Vulnerable, NT (CD) Near Threatened (Conservation Dependent), NT Near Threatened.

\begin{tabular}{|c|c|c|c|c|c|c|}
\hline Scientific name & Common name & $\begin{array}{l}\text { Australian } \\
\text { endemic }\end{array}$ & MAP status & EPBCA & IUCN & $\begin{array}{l}\text { Extinction } \\
\text { decade }\end{array}$ \\
\hline Zaglossus bruijnii & Long-beaked Echidna & NO & EX & & CR & $>1901$ \\
\hline Thylacinus cynocephalus & Thylacine & YES & EX & EX & EX & 1930 \\
\hline Chaeropus ecaudatus & Pig-footed Bandicoot & YES & EX & EX & EX & 1950 \\
\hline Perameles eremiana & Desert Bandicoot & YES & EX & EX & EX & 1960 \\
\hline Macrotis leucura & Lesser Bilby & YES & EX & EX & EX & 1960 \\
\hline Bettongia anhydra & Desert Bettong & YES & EX & & & 1950 \\
\hline Bettongia pusilla & Nullarbor Dwarf Bettong & YES & EX & & EX & 1880 \\
\hline Caloprymnus campestris & Desert Rat-kangaroo & YES & EX & EX & EX & 1930 \\
\hline Potorous platyops & Broad-faced Potoroo & YES & EX & EX & EX & $>1875$ \\
\hline Lagorchestes asomatus & Central Hare-wallaby & YES & EX & EX & EX & 1950 \\
\hline Lagorchestes leporides & Eastern Hare-wallaby & YES & EX & EX & EX & $1900 ?$ \\
\hline Macropus greyi & Toolache Wallaby & YES & EX & EX & EX & 1970 \\
\hline Onychogalea lunata & Crescent Nailtail Wallaby & YES & EX & EX & EX & 1950 \\
\hline Pteropus brunneus & Dusky Flying-fox & YES & EX & & EX & 1890 \\
\hline Nyctophilus howensis & Lord Howe Long-eared Bat & YES & EX & EX & EX & 1920 \\
\hline Pipistrellus murrayi & Christmas Island Pipistrelle & YES & EX & $\mathrm{CR}$ & CR & 2000 \\
\hline Conilurus albipes & White-footed Rabbit-rat & YES & EX & EX & EX & 1860 \\
\hline Conilurus capricornensis & Capricornian Rabbit-rat & YES & EX & & & $?$ \\
\hline Leporillus apicalis & Lesser Stick-nest Rat & YES & EX & EX & $\mathrm{CR}(\mathrm{PE})$ & $>1930$ \\
\hline Notomys amplus & Short-tailed Hopping-mouse & YES & EX & EX & EX & 1900 \\
\hline Notomys longicaudatus & Long-tailed Hopping-mouse & YES & EX & EX & EX & 1900 \\
\hline Notomys macrotis & Large-eared Hopping-mouse & YES & EX & EX & EX & 1860 \\
\hline Notomys mordax & Darling Downs Hopping-mouse & YES & EX & EX & EX & $>1840$ \\
\hline Notomys robustus & Broad-cheeked Hopping-mouse & YES & EX & & & 1870 \\
\hline Pseudomys auritus & Long-eared Mouse & YES & EX & & & 1850 \\
\hline Pseudomys glaucus & Blue-grey Mouse & YES & EX & & EX & $?$ \\
\hline Pseudomys gouldii & Gould's Mouse & YES & EX & EX & EX & $>1850$ \\
\hline Rattus macleari & Maclear's Rat & YES & EX & EX & EX & 1900 \\
\hline Rattus nativitatis & Bulldog Rat & YES & EX & EX & EX & 1900 \\
\hline Melomys rubicola & Bramble Cay Melomys & YES & $\mathrm{CR}(\mathrm{PE})$ & EN & CR & 2010 \\
\hline Crocidura trichura & Christmas Island Shrew & YES & $\mathrm{CR}(\mathrm{PE})$ & EN & & \\
\hline Lasiorhinus krefftii & Northern Hairy-nosed Wombat & YES & $\mathrm{CR}$ & EN & $\mathrm{CR}$ & \\
\hline Burramys parvus & Mountain Pygmy-possum & YES & CR & EN & $\mathrm{CR}$ & \\
\hline Gymnobelideus leadbeateri & Leadbeater's Possum & YES & $\mathrm{CR}$ & EN & EN & \\
\hline Pseudocheirus occidentalis & Western Ringtail Possum & YES & CR & VU & VU & \\
\hline Bettongia penicillata & Woylie & YES & CR & & $\mathrm{CR}$ & \\
\hline Potorous gilbertii & Gilbert's Potoroo & YES & $\mathrm{CR}$ & $\mathrm{CR}$ & $\mathrm{CR}$ & \\
\hline Pteropus natalis & Christmas Island Flying-fox & YES & CR & & & \\
\hline Zyzomys palatalis & Carpentarian Rock-rat & YES & CR & EN & $\mathrm{CR}$ & \\
\hline Zyzomys pedunculatus & Central Rock-rat & YES & $C R$ & EN & $C R$ & \\
\hline
\end{tabular}




\begin{tabular}{|c|c|c|c|c|c|}
\hline Dasyurus hallucatus & Northern Quoll & YES & EN & EN & EN \\
\hline Dasyurus viverrinus & Eastern Quoll & YES & EN & & NT \\
\hline Parantechinus apicalis & Dibbler & YES & EN & EN & EN \\
\hline Sarcophilus harrisii & Tasmanian Devil & YES & EN & EN & EN \\
\hline Myrmecobius fasciatus & Numbat & YES & EN & VU & EN \\
\hline Petaurus gracilis & Mahogany Glider & YES & EN & EN & EN \\
\hline Bettongia tropica & Northern Bettong & YES & EN & EN & EN \\
\hline Petrogale coenensis & Cape York Rock-wallaby & YES & EN & & NT \\
\hline Petrogale persephone & Proserpine Rock-wallaby & YES & EN & EN & EN \\
\hline Hipposideros inornatus & Arnhem Leaf-nosed Bat & YES & EN & & $\mathrm{VU}$ \\
\hline Arctophoca tropicalis & Subantarctic Fur Seal & NO & EN & $\mathrm{VU}$ & LC \\
\hline Balaenoptera borealis & Sei Whale & NO & EN & VU & EN \\
\hline Balaenoptera musculus & Blue Whale & NO & EN & EN & EN \\
\hline Balaenoptera physalus & Fin Whale & NO & EN & VU & EN \\
\hline Dasyuroides byrnei & Kowari & YES & VU & VU & VU \\
\hline Antechinus bellus & Fawn Antechinus & YES & VU & & LC \\
\hline Phascogale pirata & Northern Brush-tailed Phascogale & YES & VU & VU & VU \\
\hline Sminthopsis butleri & Butler's Dunnart & YES & VU & VU & VU \\
\hline Sminthopsis psammophila & Sandhill Dunnart & YES & VU & EN & EN \\
\hline Isoodon auratus & Golden Bandicoot & YES & VU & $\mathrm{VU}^{*}$ & $\mathrm{VU}$ \\
\hline Perameles bougainville & Western Barred Bandicoot & YES & VU & & EN \\
\hline Perameles gunnii & Eastern Barred Bandicoot & YES & VU & & NT \\
\hline Macrotis lagotis & Bilby & YES & VU & $\mathrm{VU}$ & $\mathrm{VU}$ \\
\hline Phascolarctos cinereus & Koala & YES & VU & $(\mathrm{VU})$ & LC \\
\hline Petauroides volans & Greater Glider & YES & VU & & LC \\
\hline Bettongia gaimardi & Southern Bettong & YES & VU & & NT \\
\hline Potorous longipes & Long-footed Potoroo & YES & VU & EN & EN \\
\hline Lagorchestes hirsutus & Mala & YES & VU & & $\mathrm{VU}$ \\
\hline Onychogalea fraenata & Bridled Nailtail Wallaby & YES & VU & EN & EN \\
\hline Petrogale lateralis & Black-footed Rock-wallaby & YES & VU & & NT \\
\hline Petrogale penicillata & Brush-tailed Rock-wallaby & YES & VU & $\mathrm{VU}$ & NT \\
\hline Petrogale sharmani & Mount Claro Rock-wallaby & YES & VU & & NT \\
\hline Setonix brachyurus & Quokka & YES & VU & VU & VU \\
\hline Lagostrophus fasciatus & Banded Hare-wallaby & YES & VU & & $\mathrm{VU}$ \\
\hline Pteropus poliocephalus & Grey-headed Flying-fox & YES & VU & VU & $\mathrm{VU}$ \\
\hline Macroderma gigas & Ghost Bat & YES & VU & & $\mathrm{VU}$ \\
\hline Rhinolophus 'intermediate' & Lesser Large-eared Horseshoe-bat & YES & VU & & \\
\hline Nyctophilus corbeni & South-eastern Long-eared Bat & YES & VU & VU & \\
\hline Chalinolobus dwyeri & Large-eared Pied Bat & YES & VU & VU & NT \\
\hline Conilurus penicillatus & Brush-tailed Rabbit-rat & NO & vu & VU & NT \\
\hline Mesembriomys gouldii & Black-footed Tree-rat & YES & VU & & NT \\
\hline Notomys aquilo & Northern Hopping-mouse & YES & VU & VU & EN \\
\hline Notomys fuscus & Dusky Hopping-mouse & YES & VU & $\mathrm{VU}$ & VU \\
\hline Pseudomys australis & Plains Mouse & YES & VU & VU & VU \\
\hline Pseudomys fieldi & Shark Bay Mouse & YES & VU & VU & VU \\
\hline Pseudomys fumeus & Smoky Mouse & YES & VU & EN & EN \\
\hline Pseudomys novaehollandiae & New Holland Mouse & YES & VU & VU & $\mathrm{VU}$ \\
\hline Pseudomys oralis & Hastings River Mouse & YES & VU & EN & VU \\
\hline
\end{tabular}




\begin{tabular}{|c|c|c|c|c|c|}
\hline Zyzomys maini & Arnhem Rock-rat & YES & VU & VU & NT \\
\hline Xeromys myoides & Water Mouse & NO & VU & VU & VU \\
\hline Neophoca cinerea & Australian Sea-lion & YES & VU & VU & EN \\
\hline Physeter macrocephalus & Sperm Whale & NO & VU & & VU \\
\hline Dasyurus geoffroii & Chuditch & YES & $\mathrm{NT}(\mathrm{CD})$ & VU & NT \\
\hline Bettongia lesueur & Boodie & YES & $\mathrm{NT}(\mathrm{CD})$ & & NT \\
\hline Petrogale xanthopus & Yellow-footed Rock-wallaby & YES & $\mathrm{NT}(\mathrm{CD})$ & & NT \\
\hline Pteropus conspicillatus & Spectacled Flying-fox & NO & NT (CD) & VU & LC \\
\hline Leporillus conditor & Greater Stick-nest Rat & YES & $\mathrm{NT}(\mathrm{CD})$ & VU & VU \\
\hline Ornithorhynchus anatinus & Platypus & YES & NT & & LC \\
\hline Dasycercus cristicauda & Crest-tailed Mulgara & YES & NT & VU & LC \\
\hline Dasyurus maculatus & Spot-tailed Quoll & YES & NT & & NT \\
\hline Pseudantechinus mimulus & Carpentarian Antechinus & YES & NT & VU & EN \\
\hline Antechinus godmani & Atherton Antechinus & YES & NT & & NT \\
\hline Phascogale calura & Red-tailed Phascogale & YES & NT & EN & NT \\
\hline Phascogale tapoatafa & Brush-tailed Phascogale & YES & NT & & NT \\
\hline Sminthopsis archeri & Chestnut Dunnart & NO & NT & & $\mathrm{DD}$ \\
\hline Sminthopsis bindi & Kakadu Dunnart & YES & NT & & LC \\
\hline Sminthopsis douglasi & Julia Creek Dunnart & YES & NT & EN & NT \\
\hline Lasiorhinus latifrons & Southern Hairy-nosed Wombat & YES & NT & & LC \\
\hline Petaurus australis & Yellow-bellied Glider & YES & NT & & LC \\
\hline Hemibelideus lemuroides & Lemuroid Ringtail Possum & YES & NT & & NT \\
\hline Pseudochirops archeri & Green Ringtail Possum & YES & NT & & LC \\
\hline Pseudochirulus cinereus & Daintree River Ringtail Possum & YES & NT & & LC \\
\hline Wyulda squamicaudata & Scaly-tailed Possum & YES & NT & & $\mathrm{DD}$ \\
\hline Potorous tridactylus & Long-nosed Potoroo & YES & NT & & LC \\
\hline Dendrolagus bennettianus & Bennett's Tree-kangaroo & YES & NT & & NT \\
\hline Dendrolagus lumholtzi & Lumholtz's Tree-kangaroo & YES & NT & & LC \\
\hline Lagorchestes conspicillatus & Spectacled Hare-wallaby & NO & NT & & LC \\
\hline Macropus bernardus & Black Wallaroo & YES & NT & & LC \\
\hline Macropus parma & Parma Wallaby & YES & NT & & NT \\
\hline Petrogale burbidgei & Monjon & YES & NT & & NT \\
\hline Petrogale concinna & Nabarlek & YES & NT & & $\mathrm{DD}$ \\
\hline Petrogale godmani & Godman's Rock-wallaby & YES & NT & & LC \\
\hline Petrogale mareeba & Mareeba Rock-wallaby & YES & NT & & LC \\
\hline Petrogale purpureicollis & Purple-necked Rock-wallaby & YES & NT & & LC \\
\hline Rhinolophus robertsi & Greater Large-eared Horseshoe-bat & YES & NT & $\mathrm{VU}^{* *}$ & \\
\hline Hipposideros cervinus & Fawn Leaf-nosed Bat & NO & NT & & LC \\
\hline Hipposideros diadema & Diadem Leaf-nosed Bat & NO & NT & & LC \\
\hline Hipposideros semoni & Semon's Leaf-nosed Bat & NO & NT & EN & $\mathrm{DD}$ \\
\hline Hipposideros stenotis & Northern Leaf-nosed Bat & YES & NT & & LC \\
\hline Saccolaimus mixtus & Cape York Sheath-tailed Bat & NO & NT & & DD \\
\hline Saccolaimus saccolaimus & Bare-rumped Sheath-tailed Bat & NO & NT & & LC \\
\hline Taphozous australis & Coastal Sheath-tailed Bat & NO & NT & & NT \\
\hline Mormopterus eleryi & Bristle-faced Free-tailed Bat & YES & NT & & \\
\hline Mormopterus norfolkensis & East-coast Free-tailed Bat & YES & NT & & LC \\
\hline Murina florium & Flute-nosed Bat & NO & NT & & LC \\
\hline
\end{tabular}




\begin{tabular}{|c|c|c|c|c|}
\hline Nyctophilus sherrini & Tasmanian Long-eared Bat & YES & NT & \\
\hline Falsistrellus mackenziei & Western False Pipistrelle & YES & NT & \\
\hline Mastacomys fuscus & Broad-toothed Rat & YES & NT & \\
\hline Mesembriomys macrurus & Golden-backed Tree-rat & YES & NT & VU \\
\hline Notomys cervinus & Fawn Hopping-mouse & YES & NT & \\
\hline Pseudomys calabyi & Kakadu Pebble-mouse & YES & NT & \\
\hline Pseudomys occidentalis & Western Mouse & YES & NT & \\
\hline Pseudomys shortridgei & Heath Mouse & YES & NT & VU \\
\hline Uromys hadrourus & Pygmy White-tailed Rat & YES & NT & \\
\hline Dugong dugon & Dugong & NO & NT & \\
\hline Canis lupus dingo*** & Dingo & $(\mathrm{NO})$ & NT & \\
\hline Mirounga leonina & Southern Elephant Seal & NO & NT & VU \\
\hline Eubalaena australis & Southern Right Whale & NO & NT & EN \\
\hline Orcaella heinsohni & Australian Snubfin Dolphin & NO & NT & \\
\hline Sousa chinensis $* * * *$ & Indo-Pacific Hump-backed Dolphin & NO & NT & \\
\hline
\end{tabular}

* For both subspecies I. a. auratus and I. a barrowensis; ${ }^{* *}$ as R. p. 'large form'; ${ }^{* * *}$ contested taxonomy, with some recent treatment as an endemic Australian species, C. dingo (Crowther et al. 2014); **** now recognised as S. sahulensis Australian Hump-backed Dolphin, but with unchanged conservation status in Australian range.

in decline include changed fire regimes, habitat loss and fragmentation, and habitat degradation due to introduced livestock and feral herbivores. The role of introduced disease is generally largely unknown, but there is some strong circumstantial evidence for some species (Abbott 2006), and a particularly unusual (and currently untreatable) disease is entirely responsible for the current rapid decline of Tasmanian devil Sarcophilus harrisii, the largest remaining marsupial carnivore (McCallum et al. 2009). Climate change has not yet had a major impact, but is projected to cause substantial declines for some species in some areas (particularly in tropical mountain rainforests and alpine areas; Williams et al. 2003).

In many cases, individual mammal species are, or have been, affected by multiple threatening factors operating synergistically at any one location, or differently across different parts of the species' range, or at different times. For example, in northern Australia, recent research has indicated that predation by feral cats has greater impact in areas exposed to more frequent and/or extensive fires (McGregor et al. 2014). In south-western Australia, concerted management action has led to some benefit through reduction in the abundance of the European red fox, but that change in abundance has now led to increased impacts of predation by feral cats (Marlow et al. 2015). Many species now face multiple threats: for example, the koala Phascolarctos cinereus is affected by disease (including Chlamydia and a retrovirus), predation by feral (and stray) dogs, timber harvesting, changed fire regimes, road traffic, habitat loss and fragmentation, drought and days of extreme heat.

Largely because of the identification and effective management of factors responsible for decline, some threatened terrestrial mammal species have shown some recovery over the last few decades. The most notable of these successes have involved translocation of individuals from remnant populations to islands or to intensively managed mainland areas (notably predator-proof exclosures) and broad-scale control of foxes (Woinarski et al. 2014). Notwithstanding these efforts, the conservation status of only six terrestrial mammal species was considered to have been improved over the last 20 years.

For Australian marine mammals, the conservation status and outlook is far less clear. Only one species, the Australian sea lion Neophoca cinerea, is endemic to Australian waters. Of 58 species recorded from Australian waters, the information base for conservation parameters in these waters is so meagre for 35 species that no categorisation other than Data Deficient is justifiable. Many of these species may be imperilled or otherwise declining, but the evidence base is inadequate to 
demonstrate this. Several previously highly threatened species, most notably the Humpback Whale Megaptera novaeangliae, are recovering (and their conservation status was assessed as improving) in Australian waters following cessation of hunting (whaling or sealing). However, a wide range of threats - notably mortality from fisheries interactions, pollution, acoustic disturbance and habitat degradation - continues to affect most marine mammals in Australian waters, and it is likely that climate change and ocean acidification will lead to further declines.

\section{Discussion}

This review highlighted the disintegration of a diverse and highly distinctive mammal fauna over a period of little more than two centuries, and - alarmingly - concluded that the rate of loss is unabated. The number of extinct species is appreciably higher than previously recognised under Australian national legislation (where 20 Australian mammal species are currently listed as extinct) or from the most recent global assessment (22 Australian mammal species are currently categorised as extinct in the IUCN Red List). No other country has suffered such a high number of mammal extinctions over recent centuries. Many of the now-extinct mammal species were widespread, abundant and had broad habitat ranges at the time of European settlement (Hanna and Cardillo 2013): they were unlikely candidates for extinction.

The Australian mammal fauna has shown a susceptibility to the impacts of introduced species, particularly two predators, that is characteristic of the frailty of island biodiversity generally (Alcover et al. 1998). To some extent, conservation managers have now developed and implemented approaches that provide some control for one of these introduced predators, the European red fox, and sustained and large-scale conservation effort directed at this threat is achieving some impressive reversals of previous declining trends for some threatened species (Morris et al. 2003). But to date there has been no successful landscape-scale control of the almost pervasive feral cat. The only remedial actions that have had some success against this threat have been localised and expensive predator-proof exclosures or translocations to cat-free islands.

Other intensive remedial conservation management has helped stave off extinctions for some high profile Australian mammal species faced with a range of sometimes idiosyncratic threats (McCallum et al. 2009). However, much of the current decline in the Australian mammal fauna involves poorlyknown low profile terrestrial species occurring in remote and ostensibly little modified areas, or is occurring largely unreported but incrementally for marine species, and there is little management attention to, or public awareness of the plight of, these species. Currently, there is insufficient knowledge of the status of many of these species, of the extent to which different threats are driving decline, or of management options to counter these threats. There are insufficient resources to manage the range of threats, and there is no coherent or consolidated response to address this crisis. Without major change in resourcing, management, policy and accountability, it is highly likely that this mammal fauna will continue to substantially erode.

\section{Acknowledgements}

Our review was greatly informed by the inputs of more than 200 experts familiar with the Australian mammal fauna, and was supported in part by the Australian Wildlife Conservancy, Norman Wettenhall Foundation, Australian Department of the Environment, and the North Australian Hub of the National Environmental Research Program. 


\title{
Resumen
}

\begin{abstract}
Introducción: En este trabajo se presenta un resumen de los resultados de una reciente revisión exhaustiva del estado de conservación de todas las especies y subespecies de la tierra y de mamíferos marinos Australianos. Desde la fecha histórica de la colonización Europea de Australia (1788), al menos 28 de las 272 especies de mamíferos terrestres endémicos australianos han quedado extintos.
\end{abstract}

Resultados y Discusión: Las extinciones se han producido a un ritmo más o menos constante de una a dos especies por década desde la década de 1840, con una tasa continua sin cesar. Otras 55 especies de la fauna original ahora se encuentran amenazadas, y otras 42 están en la categoría de "Casi Amenazadas". Aunque muchos factores han contribuido a esta disminución y extinción, y la gama de amenazas varía entre las distintas especies, la amenaza que más ha tenido (y sigue teniendo) un impacto perjudicial es la depredación por el gato introducido Felis catus y la zorra roja Europeo Vulpes vulpes. Ha habido un cierto éxito a gran escala en el manejo de la zorra, pero la amenaza planteada por los gatos salvajes sigue creciendo y en gran parte sin limites. Para las 55 especies presentes en las aguas marinas de Australia, la base de la información es en su mayoría demasiado escasa para evaluar el estado de conservación de otra forma que como "Datos Insuficientes". Para la fauna de mamíferos australianos en general, el esfuerzo actual de manejo de conservación es insuficiente, con una tendencia para el continuo declive de muchas especies- por ejemplo, de 49 especies cuyo estado de conservación cambió durante el período 1992-2012, 38 mostraban un estado de conservación en deterioro dmientras que sólo 11 tenían mejora de la situación.

Palabras Clave: amenazada, conservación, gato silvestre, extinto , mamífero marino, marsupial , depredación, translocación, zorra roja

\section{References}

Аввотт, I. 2006. Mammalian faunal collapse in Western Australia, 1875-1925: the hypothesised role of epizootic disease and a conceptual model of its origin, introduction, transmission, and spread. Australian Zoologist 33:530-561.

Alcover, J. A., A. Sans, ANd M. Palmer. 1998. The extent of extinctions of mammals on islands. Journal of Biogeography 25:913-918.

Baker, A. M., T. Y. Mutton, And H. B. Hines. 2013. A new dasyurid marsupial from Kroombit Tops, south-east Queensland, Australia: the Silver-headed Antechinus, Antechinus argentus sp. nov.(Marsupialia: Dasyuridae). Zootaxa 3746:201-239.

Baker, A. M., T. Y. Mutton, H. B. Hines, and S. Van Dyck. 2014. The Black-tailed Antechinus, Antechinus arktos sp. nov.: a new species of carnivorous marsupial from montane regions of the Tweed Volcano caldera, eastern Australia. Zootaxa 3765:100-133.

BakeR, A. M., T. Y. Mutton, And S. Van Dyck. 2012. A new dasyurid marsupial from eastern Queensland, Australia: the Buff-footed Antechinus, Antechinus mysticus sp. nov. (Marsupialia: Dasyuridae). Zootaxa 3515:1-37.

Bannister, J. L., C. M. Kemper, and R. M. Warneke. 1996. The action plan for Australian cetaceans. Australian Nature Conservation Agency.

Bradshaw, C. J. A. 2012. Little left to lose: deforestation and forest degradation in Australia since European colonization. Journal of Plant Ecology 5:109-120.

Burbidge, A. A. 1999. Conservation values and management of Australian islands for non-volant mammal conservation. Australian Mammalogy 21:67-74.

Burbidge, A. A., ANd N. L. Mckenzie. 1989. Patterns in the modern decline of Western Australia's vertebrate fauna: causes and conservation implications. Biological Conservation 50:143-198.

Burbidge, A. A., N. L. Mckenzie, K. E. C. Brennan, J. C. Z. Woinarski, C. R. Dickman, A. Baynes, G. Gordon, P. W. Menkhorst, and A. C. Robinson. 2009. Conservation status and biogeography of Australia's terrestrial mammals. Australian Journal of Zoology 56:411-422.

Cardillo, M., And L. Bromham. 2001. Body size and risk of extinction in Australian mammals. Conservation Biology 15:1435-1440. 
Chisholm, R., and R. Taylor. 2007. Null-hypothesis significance testing and the critical weight range for Australian mammals. Conservation Biology 21:1641-1645.

Crowther, M. S., M. Fillios, N. Colman, And M. Letnic. 2014. An updated description of the Australian dingo (Canis dingo Meyer, 1793). Journal of Zoology 293:192-203.

Duncan, A., G. B. Baker, And N. Montgomery. 1999. The action plan for Australian bats. Environment Australia. Canberra, Australia.

Fisher, D. O., C. N. Johnson, M. J. Lawes, S. A. Fritz, H. Mccallum, S. P. Blomberg, J. Vanderwal, B. Abbott, A. Frank, S. Legge, M. Letnic, C. R. Thomas, A. Fisher, I. J. Gordon, and A. Kutt. 2014. The current decline of tropical marsupials in Australia: is history repeating? Global Ecology and Biogeography 23:181-190.

Garnett, S. T., And G. M. Crowley. 2000. The Action Plan for Australian Birds 2000. Environment Australia. Canberra, Australia.

Garnett, S. T., J. K. Szabo, And G. Dutson. 2011. The action plan for Australian birds 2010. CSIRO Publishing. Collingwood.

Hanna, E., and M. Cardillo. 2013. A comparison of current and reconstructed historic geographic range sizes as predictors of extinction risk in Australian mammals. Biological Conservation 158:196-204.

Hoffmann, M., J. L. Belant, J. S. Chanson, N. A. Cox, J. Lamoreux, A. S. L. Rodrigues, J. Schipper, and S. N. Stuart. 2011. The changing fates of the world's mammals. Philosophical Transactions of the Royal Society B: Biological Sciences 366: 2598-2610.

Holt, B. G., J.-P. Lessard, M. K. Borregaard, S. A. Fritz, M. B. Araújo, D. Dimitrov, P.-H. Fabre, C. H. Graham, G. R. Graves, K. A. Jønsson, D. Nogués-Bravo, Z. Wang, R. J. Whittaker, J. Fejdså avnd C. Rahbek. 2013. An update of Wallace's zoogeographic regions of the World. Science 339:74-78.

IucN. 2001. Red List Categories and Criteria, version 3.1. Gland Switzerland and Cambridge, U.K.

Iucn Standards, and Petitions Subcommittee. 2013. Guidelines for Using the IUCN Red List Categories and Criteria. Version 10. Gland, Switzerland.

Jackson, S. M., ANd C. P. Groves. in Press. Systematics and Taxonomy of Australian Mammals. CSIRO Publishing. Melbourne.

Jefferson, T. A., and H. C. Rosenbaum. 2014. Taxonomic revision of the humpback dolphins (Sousa spp.), and description of a new species from Australia. Marine Mammal Science 30:1494-1541.

Johnson, C. 2006. Australia's mammal extinctions: a 50,000 year history. Cambridge University Press. Port Melbourne.

Johnson, C. N., AND J. L. IsaAC. 2009. Body mass and extinction risk in Australian marsupials: the 'Critical Weight Range' revisited. Austral Ecology 34:35-40.

LeE, A. K. 1995. The action plan for Australian rodents. Australian Nature Conservation Agency. Canberra, Australia.

Marlow, M. J., N. D. Thomas, A. A. E. Williams, B. Macmahon, J. Lawson, Y. Hitchen, J. Angus, and O. Berry, 2015. Cats (Felis catus) are more abundant and are the dominant predator of woylies (Bettongia penicillata) after sustained fox (Vulpes vulpes) control. Australian Journal of Zoology

Maxwell, S., A. A. Burbidge, And K. Morris. 1996. The 1996 action plan for Australian marsupials and monotremes. Wildlife Australia. Canberra, Australia.

Mccallum, H., M. Jones, C. Hawkins, R. Hamede, S. Lachish, D. L. Sinn, N. Beeton, and B. Lazenby. 2009. Transmission dynamics of Tasmanian devil facial tumor disease may lead to disease-induced extinction. Ecology 90:3379-3392.

Mcgregor, H. W., S. LegGe, M. E. Jones, And C. N. Johnson. 2014. Landscape management of fire and grazing regimes alters the fine-scale habitat utilisation by feral cats. PloS One 9: e109097.

Mckenzie, N. L., A. A. Burbidge, A. Baynes, R. N. Brereton, C. R. Dickman, G. Gordon, L. A. Gibson, P. W. Menkhorst, A. C. Robinson, M. R. Williams, and J. C. Z. Woinarski. 2007. Analysis of factors implicated in the recent decline of Australia's mammal fauna. Journal of Biogeography 34:597-611. 
Morris, K., B. Johnson, P. Orell, G. Gaikhorst, A. Wayne, and D. Moro. 2003. Recovery of the threatened chuditch (Dasyurus geoffroii): a case study. Pp. 435-451 in Predators with pouches: the biology of carnivorous marsupials. (Jones, M., C. Dickman, and M. Archer, eds.) CSIRO Publishing. Melbourne, Australia.

Murphy, B. P., AND H. F. Davies. 2014. There is a critical weight range for Australia's declining tropical mammals. Global Ecology and Biogeography 23:1058-1061.

Reardon, T., N. L. Mckenzie, S. Cooper, B. Appleton, S. Carthew, and M. Adams. 2014. A molecular and morphological investigation of species boundaries and phylogenetic relationships in Australian free-tailed bats Mormopterus (Chiroptera: Molossidae). Australian Journal of Zoology 62:109-136.

Schipper, J., J. S. Chanson, F. Chiozza, N. A. Cox, M. Hoffmann, V. Katariya, J. Lamoreux, A. S. L. Rodrigues, S. N. Stuart, H. J. Temple, J. Baillie, L. Boitani, L. T.E.JR., R. A. Mittermeier, A. T. Smith, D. Absolon, J. M. Aguiar, G. Amori, N. Bakkour, R. Baldi, R. J. Berridge, J. Bielby, P. A. Black, J. J. Blanc, T. M. Brooks, J. A. Burton, T. M. Butynski, G. Catullo, R. Chapman, Z. Cokeliss, B. Collen, J. Conroy, J. G. Cooke, G. A. B. Da Fonseca, A. E. Derocher, H. T. Dublin, J. W. Duckworth, L. Emmons, R. H. Emslie, M. Festa-Bianchet, M. Foster, S. Foster, D. L. Garshelis, C. Gates, M. Gimenez-Dixon, S. Gonzalez, J. F. Gonzalez-Maya, T. C. Good, G. Hammerson, P. S. Hammond, D. Happold, M. Happold, J. Hare, R. B. Harris, C. E. Hawkins, M. Haywood, L. R. Heaney, S. Hedges, K. M. Helgen, C. Hilton-Taylor, S. A. Hussain, N. Ishil, T. A. Jefferson, R. K. B. Jenkins, C. H. Johnston, M. Keith, J. Kingdon, D. H. Knox, K. M. Kovacs, P. Langhammer, K. Leus, R. Lewison, G. Lichtenstein, L. F. Lowry, Z. Macavoy, G. M. Mace, D. P. Mallon, M. Masi, M. W. Mcknight, R. A. Medellín, P. Medici, G. Mills, P. D. Moehlman, S. Molur, A. Mora, K. Nowell, J. F. Oates, W. Olech, W. R. L. Oliver, M. Oprea, B. D. Patterson, W. F. Perrin, B. A. Polidoro, C. Pollock, A. Powel, Y. Protas, P. Racey, J. Ragle, P. Ramani, G. Rathbun, R. R. Reeves, S. B. Reilly, J. E. I. Reynolds, C. Rondinini, R. G. Rosell-Ambal, M. Rulli, A. B. Rylands, S. Savini, C. J. Schank, W. Sechrest, C. Self-Sullivan, A. Shoemaker, C. Sillero-Zubiri, N. De Silva, D. E. Smith, C. Srinivasulu, P. J. Stephenson, N. Van Strien, B. K. Talukdar, B. L. Taylor, R. Timmins, D. G. Tirira, M. F. Tognelli, K. Tsytsulina, L. M. Veiga, J.-C. Vié, E. A. Williamson, S. A. Wyatt, Y. Xie, and B. E. Young. 2008. The status of the World's land and marine mammals: diversity, threat, and knowledge. Science 322:225-230.

Shaughnessy, P. D. 1999. The action plan for Australian seals. Environment Australia. Canberra, Australia.

Williams, S. E., E. E. Bolitho, AND S. Fox. 2003. Climate change in Australian tropical rainforests: an impending environmental catastrophe. Proceedings of the Royal Society of London B. Biological Sciences 264:1887-1892.

Woinarski, J. C. Z., A. A. Burbidge, And P. L. Harrison. 2014. The Action Plan for Australian Mammals 2012. CSIRO Publishing. Melbourne.

Woinarski, J. C. Z., S. Legge, J. A. Fitzsimons, B. J. Traill, A. A. Burbidge, A. Fisher, R. S. C. Firth, I. J. Gordon, A. D. Griffiths, C. N. Johnson, N. L. Mckenzie, C. Palmer, I. Radford, B. Rankmore, E. G. Ritchie, S. WARD, AND M. ZIEMBICKI. 2011. The disappearing mammal fauna of northern Australia: context, cause, and response. Conservation Letters 4:192-201.

Summited: September 30, 2014

Review: January 10, 2015

Accepted: January 28, 2015

Associated editor: Jesus Maldonado 